\title{
The diversity and abundance of phytoplankton and benthic diatoms in varying environmental conditions in Kok River, Chiang Rai, Thailand as bio-indicators of water quality
}

\author{
TIPPAWAN PRASERTSIN ${ }^{1,}$, KRITTAWIT SUK-UENG ${ }^{2}$, KITTIYA PHINYO $^{3}$, EKKACHAI YANA ${ }^{4}$ \\ ${ }^{1}$ Biological Science Program, Faculty of Science and Technology, Chiang Rai Rajabhat University. Chiang Rai 57100, Thailand. \\ Tel.:+66-85-459-4633, Fax.: +66-53-702758, "email: prasertsintippawan@ gmail.com \\ ${ }^{2}$ Energy and Environment Program, Faculty of Science and Technology, Chiang Rai Rajabhat University. Chiang Rai 57100, Thailand \\ ${ }^{3}$ Department of Biology, Faculty of Science, Chiang Mai University, Chiang Mai 50200, Thailand \\ ${ }^{4}$ Environmental Science and Technology Program, Faculty of Science, Lampang Rajabhat University. Lampang 52100, Thailand
}

Manuscript received: 13 January 2021. Revision accepted: 21 March 2021.

\begin{abstract}
Prasertsin T, Suk-ueng K, Phinyo K, Yana E. 2021. The diversity and abundance of phytoplankton and benthic diatoms in varying environmental conditions in Kok River, Chiang Rai, Thailand as bio-indicators of water quality. Biodiversitas 22: $1853-1862$. The study of living organisms was important information for bio-indicators which was utilized to assess the quality of the environment. In the river ecosystem, the algae with which one organism was interesting accordingly considered the relationship of 2 groups algae, including phytoplankton and benthic diatoms and the physical-chemical parameters of Kok River Chiang Rai, Thailand. Phytoplankton and benthic diatoms were collected from five sampling sites along the Kok River during January (cool dry), March (summer), and June (rainy season) 2018, and assessed as bio-indicators to monitor environmental factors that represent water quality across varying conditions and periods. Fifty-seven species of phytoplankton and thirty-nine species of benthic diatoms were found. The phytoplankton mainly belonged to the phylum Bacillariophyta (diatom group). Physical and chemical factors affecting the dominant phytoplankton and benthic diatom species were subjected to canonical correspondence analysis (CCA). Results showed that planktonic and benthic Gomphonema lagenula positively correlated with ammonium nitrogen, recording highest abundance during the rainy season. Abundance of Achnanthidium straubianum, a planktonic and benthic diatom, and Navicula cincta negatively correlated with river velocity and water conductivity, with lowest numbers during the rainy season. Water trophic status evaluated from the main parameters of the AARL-PC score indicated that during the cool dry and summer seasons all sampling sites were classified as mesotrophic, while during the rainy season they were classified as meso-eutrophic. The combination of phytoplankton and benthic diatom can be used as bio-indicators of water quality in the Kok River and other freshwater ecosystems.
\end{abstract}

Keywords: AARL-PC score, bioindicator, correlation, environmental factor, standing water

\section{INTRODUCTION}

Algae play a significant role in global ecology and ecosystem functioning as important oxygen-producing communities in aquatic environments. Based on habitats, algae can be divided into two groups, i.e. phytoplankton and benthic algae. Phytoplankton is autotrophic organism that lives near the water surface where there is sufficient light to support photosynthesis. Among the more important groups of phytoplankton are the diatoms, cyanobacteria, green algae, euglenoids and dinoflagellates. On the other hand, benthic algae are those attached to all kinds of substrata including rocks, mud, organic and inorganic particles, macrophytes and other living organisms (John et al. 2011).

Two different kinds of communities are usually recognized as microalgae and macroalgae (Lobo et al. 2016). A major group of benthic microalgae comprises the diatoms. Diatoms are abundant in marine and freshwater habitats which in all freshwater habitats including standing and running waters and planktonic and benthic habitats. They dominate both the biomass and biological diversity of the microscopic flora in many aquatic ecosystems. Diatoms inhabit a broad array of habitats but many have specific habitat requirements and have been used as freshwater environment bioassessment indicators to monitor long-term changes in ecological conditions (Blanco and Ector 2009).

Algae is sensitive to changes in their surroundings, as such total algal biomass and certain species are often used as indicators of water quality (Omar 2010). Algae have a long history of use as bio-indicators to determine water quality. The uses of phytoplankton as indicators of water quality were investigated by Peruma et al. (2009); Stepankova et al. (2012); Järvinen et al. (2012); Borics et al. (2014). In Thailand, ecological studies on water quality and its effects on the distribution and diversity of phytoplankton were conducted by Peerapornpisal et al. (2004); Prasertsin et al. (2018) while the applicability of benthic diatom indices to assess river water quality was investigated by Pekthong (2008); Suphan and Peerapornpisal (2010); Yana et al. (2013); Leelahakriengkrai and Peerapornpisal (2014); Nakkaew et al. (2015); Leelahakriengkrai and Kunpradid (2018). While such studies present the use algae as bioindicators of water quality, each study focused on a particular group of algae, either the phytoplankton or the benthic diatoms. No studies in Thailand have integrated 
both groups of algae used as bio-indicators.

The Kok River is the main river in Chiang Rai Province, Thailand and one of the tributaries of the Mekong. The characteristics of the river vary from lentic to fast-flowing stretches and this habitat diversity attracts many animals, plants and microorganisms that can be used as bio-indicators. This study aimed to examine the diversity, distribution and abundance of phytoplankton and benthic diatoms, and to investigate their relationships with environmental factors in Kok River. Our data present the first report in Thailand that combines phytoplankton and benthic diatom data as indicators of water quality in the Kok River.

\section{MATERIALS AND METHODS}

\section{Study area and period}

The Kok River is the main waterway in Chiang Rai Province as a tributary of the Mekong. From its source in Myanmar, the Kok River runs eastward through Chiang Rai and joins the Mekong at Bann Sobkok, Chiang San District. Total length of the river is $290 \mathrm{~km}$, with $114 \mathrm{~km}$ running across Chiang Rai Province (Pekthong 2008). Five sampling sites along the Kok River were selected, based on the properties of the water body and environmental impact. Details and locations of each sampling site are shown in Table 1 and Figure 1. Phytoplankton, benthic diatoms and physicochemical water quality properties were determined during January (cool dry), March (summer) and June (rainy season) 2018.

\section{Collection and identification of phytoplankton}

Twenty liters of water samples were collected from each sampling site along the Kok River and filtered using a $10 \mu \mathrm{m}$ mesh size plankton net. The samples were preserved by adding $0.7 \mathrm{~mL}$ of Lugol's solution to $100 \mathrm{~mL}$ of the sample (Eaton et al. 2005), observed under a light microscope and photographed using an Olympus Normaski Microscope. The phytoplankton specimens were identified based on relevant characteristics, such as color, cell-size, colony or filament, shape of the chloroplast, number and position of the flagella with or without the spine, as well as details of the granular characteristics of the cell wall (John et al. 2011; Prasertsin et al. 2014; Phinyo et al. 2017).

Table 1. Sampling sites locations and character of water types along the Kok River, Chiang Rai Province, Thailand

\begin{tabular}{|c|c|c|}
\hline Sampling site & Location & $\begin{array}{l}\text { Character of } \\
\text { water type }\end{array}$ \\
\hline Site 1 Huay Mark Liam & Doi Hang Sub-district, (1957'49.4"N 9940'40.1"E) & Fast-flowing \\
\hline Site 2 Had Chiang Rai & Rop Wiang Sub-district, $\left(19^{\circ} 55^{\prime} 03.8^{\prime \prime N} 99^{\circ} 47^{\prime} 37.2^{\prime \prime E}\right)$ & Fast-flowing \\
\hline Site 3 Kok River bridge & Ban Rong Suea Ten, Rim Kok Sub-district, Mueang (1955'18.4"N 9950'45.6"E) & Almost still \\
\hline Site 4 Chaloem Phrakiat 1 & Ban Kwae Wai, Rop Wiang Sub-district, (1955'29.8"N 9951'46.5"E) & Almost still \\
\hline Site 5 Fai Chiang Rai & Ban PA Yang Mon, Rim Kok Sub-district, (1955'31.4"N 9953'49.9"E) & Slow-flowing \\
\hline
\end{tabular}

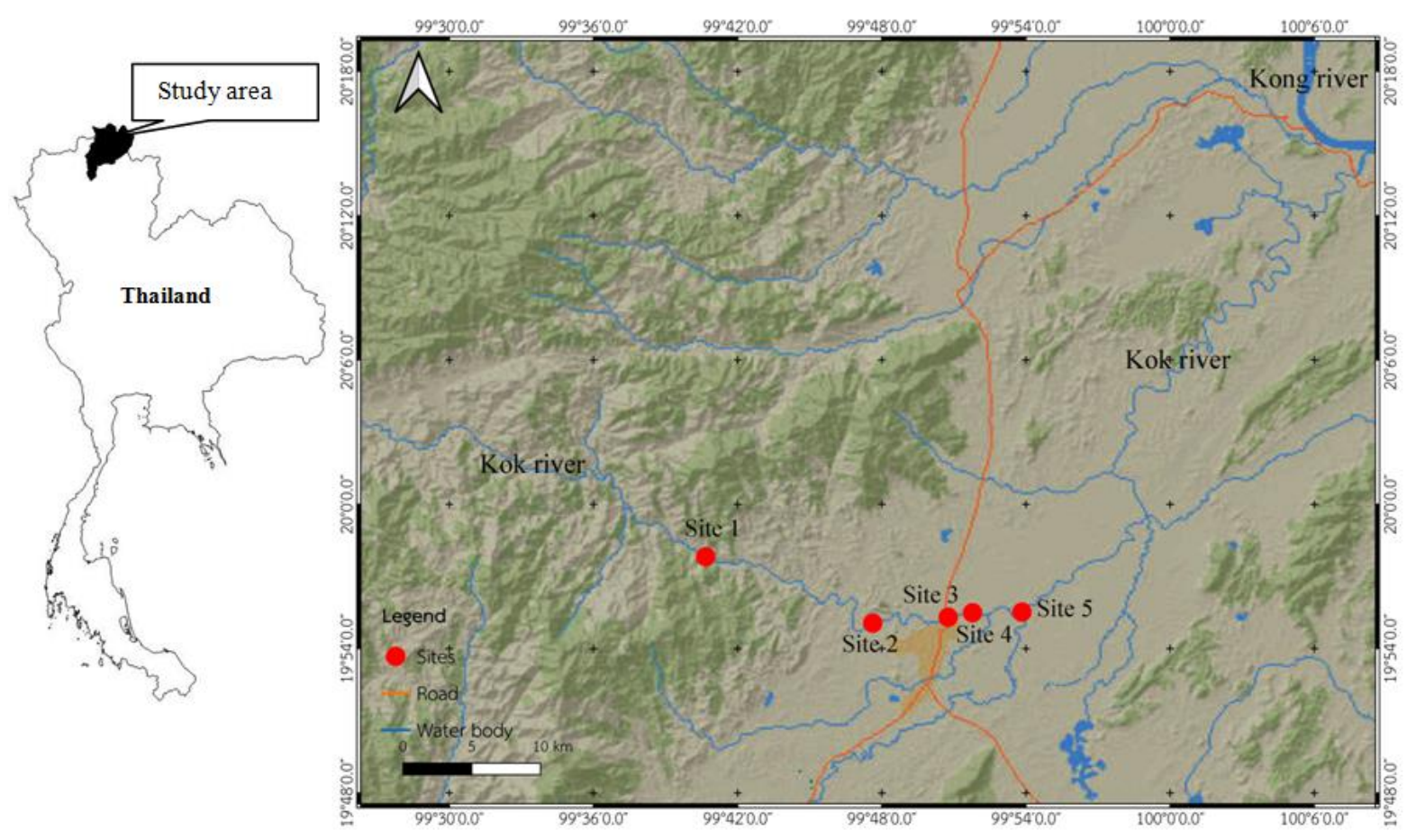

Figure 1. Map of the location of the study areas and sampling sites along the Kok River, Chiang Rai Province, Thailand 
Cell enumeration of the phytoplankton species was assessed by the Lackey drop method (Phinyo et al. 2017). This is a simple method that is used to obtain results when studying the density of plankton populations. Dominance (Y) of a species was calculated by the following equation (Yang et al. 2016):

$\mathrm{Y}=\underline{\mathrm{ni}}$.fi
Where:
$\mathrm{ni}=$ the abundance of species $\mathrm{i}$
$\mathrm{fi}=$ the occurrence frequency of species $\mathrm{i}$
$\mathrm{N}=$ the total abundance

The occurrence frequency of a species refers to the proportion of the number of stations reporting its occurrence to the total number of sampling stations. A dominant species was defined if $\mathrm{Y}$ was greater or equal to 0.02 .

\section{Collection and identification of benthic diatoms}

Benthic diatom samples were collected by scratching the substrate surface of cobbles and gravel with a toothbrush. The samples were placed in plastic boxes and fixed with Lugol's solution on site. Diatom samples were then taken into the laboratory and cleaned following the concentrated acid digestion method. The collected samples were boiled in hot nitric acid for $15 \mathrm{~min}$, followed by hydrogen peroxide for $15 \mathrm{~min}$ before rinsing with distilled water (Yana and Mayama 2015). For light microscopy (LM), cleaned samples were mounted in Naphrax (SPI Supplies, West Chester, USA). All diatom specimens in each slide were counted, identified and photographed using an Olympus Normaski Microscope with a $\times 100$ oil immersion objective. Taxonomy and nomenclature were determined according to Lange-Bertalot (2001); Yana et al. (2013); Nakkaew et al. (2015)

\section{Determination of physical and chemical properties of water}

Determination of the relevant physical and chemical properties of the river water was conducted at each sampling site by measuring air temperature, water temperature, velocity, $\mathrm{pH}$, conductivity and dissolved oxygen (DO). Water samples were collected in polyethylene bottles and kept in a cool box at $5-7^{\circ} \mathrm{C}$ for laboratory analyses of alkalinity, biochemical oxygen demand (BOD), nitrate nitrogen, ammonium nitrogen and soluble reactive phosphorus (SRP) (Eaton et al. 2005).

The trophic status of water was evaluated from the main parameters (i.e. conductivity, DO, BOD, ammonium nitrogen, nitrate nitrogen and soluble reactive phosphorus) by the Applied Algal Research Laboratory-Physical and Chemical score: AARL PC-score (Peerapornpisal et al. 2004).

\section{Relationship between phytoplankton content and physical and chemical factors}

Comparison between parameters in each season was estimated using ANOVA, followed by Tukey's post hoc test at $\mathrm{p}<0.05$. Relationships between phytoplankton, benthic diatoms and physical and chemical factors were explored by canonical correspondence analysis (CCA) using MVSP (Multi-Variate Statistical Package for Windows ver. 3.22. The result was presented as a CCA plot.

\section{RESULTS AND DISCUSSION}

\section{Distribution of phytoplankton and benthic diatoms in the Kok River}

Four Phyla containing 57 species of phytoplankton were found in the Kok River. The most diverse Phylum was Chlorophyta (31 species), followed by Bacillariophyta (18 species), Cyanobacteria (7 species) and Euglenozoa (1 species) (Table 2). All phytoplankton species found in the Kok River were acknowledged as common species that are typically found in standing water throughout Thailand (Pollution Control Department 2010). Each species showed different distribution at the sampling sites. All species in the Phyla Chlorophyta, Cyanobacteria and Euglenozoa had the highest distribution in almost still and slowly flowing water (Sites 1, 2 and 3), while Phylum Bacillariophyta in planktonic form was found at all five sampling sites. Each species was recorded at a different percentage of relative abundance during each season. Relative abundance can be used to consider the dominant species (Yang et al. 2016). Dominant phytoplankton in the Kok River was from the Phylum Bacillariophyta (diatom group). These were found at all sampling sites and included Achnanthidium straubianum (Lange-Bertalot) Lange-Bertalot, Cymbella tugidula Grunow, Gomphonema lagenula Kützing and Navicula cincta (Ehrenberg) Ralfs.

Thirty-nine species of benthic diatoms were found in the Kok River (Table 3 and Figure 2). Most were common species found in lotic ecosystems throughout Thailand (Pekthong 2008; Yana et al. 2013; Nakkaew et al. 2015; Leelahakriengkrai and Kunpradid 2018). The majority of benthic diatoms were pennate $(97 \%)$, while the remaining $3 \%$ were centric diatoms. This finding concurred with Leelahakriengkrai and Peerapornpisal (2010) who noted that pennate diatoms dominated freshwater bodies, whereas centric diatoms were more abundant in marine ecosystems. Achnanthidium straubianum (Lange-Bertalot), Cocconeis placentula Ehrenberg, Gomphonema lagenula Kützing, Planothidium frequentissimum (Østrup) Lange-Bertalot, Nitzschia inconspicua Grunow, Nitzschia palea (Kützing) W.Smith and Nitzschia supralitorea Lange-Bertalot were the seven dominant species of benthic diatoms recorded in the Kok River. Achnanthidium straubianum (Lange-Bertalot) Lange-Bertalot and Gomphonema lagenula Kützing were found in planktonic and benthic diatoms, agreeing with (Guiry and Guiry (2020), who reported that these two species occurred in all freshwater biospheres including standing and flowing waters and planktonic and benthic habitats. Cocconeis placentula Ehrenberg, Cymbella tugidula Grunow and Nitzschia palea (Kützing) W.Smith were the dominant species in the Kok River. This result concurred with Leelahakriengkrai and Peerapornpisal (2011) who indicated that these three species were the 
dominant benthic diatoms in all the main rivers of Thailand. Cocconeis placentula Ehrenberg, Cymbella tugidula Grunow, Nitzschia palea (Kützing) W.Smith, Planothidium lanceolatum (Brébisson ex Kützing) LangeBertalot, Navicula cryptotenella Lange-Bertalot in Krammer \& Lange-Bertalot, Achnanthidium exiguum (Grunow) Czarnecki, Seminavis strigose (Hustedt) Danieledis \& Economou-Amilli in Danielidis \& D.G.Mann, and Navicula germainii J.H.Wallace were most abundant species found in the Ping River Chiang Mai Province, Thailand (Leelahakriengkrai and Kunpradid 2018).

\section{Water quality based on physical and chemical properties}

Different values of physical and chemical parameters at each sampling site along the Kok River were recorded during the cool dry, summer and rainy seasons (Table 4). Air and water temperatures ranged between $22.00 \pm 0.00$ and $31.00 \pm 0.00{ }^{\circ} \mathrm{C}$ and $20.67 \pm 0.29$ and $27.00 \pm 0.00{ }^{\circ} \mathrm{C}$, respectively. The highest value occurred during the summer season at site 4 , while the lowest was recorded during the cool dry season at site 1 . Water turbidity gave diverse readings, ranging between $16.73 \pm 0.47$ and $537.00 \pm 35.59$ NTU. Highest value occurred during the rainy season at site 2 , while the lowest was recorded during the summer season at site 4 . Turbidity was reported at less than 10 NTU in some clean and clear headwater streams. Differences in turbidity were recorded throughout the sampling period, with maximum turbidity value at every sampling site during the rainy season. Alkalinity in the Kok River ranged between $56.67 \pm 4.16$ and $142.67 \pm 4.62 \mathrm{mg} \mathrm{L}^{-1}$. The value of alkalinity in the cool dry season indicated low impact of effluents, while during the summer and rainy seasons the impact of effluents was higher. Natural water alkalinity ranges between 50 and $100 \mathrm{mg} \mathrm{L}^{-1}$ and is frequently less than $100 \mathrm{mg} \mathrm{L}^{-1}$ in clean resources. The $\mathrm{pH}$ in the Kok River ranged between $6.42 \pm 0.07$ and $7.33 \pm 0.15$. On average, all sampling sites were neutral throughout the summer and rainy seasons and slightly acid during the cool dry season. Water at all sampling sites water was livable for living organisms and suitable for human consumption as it did not exceed the quality standard (6-9) of surface water (Simachaya 2000; Evans et al. 2012). Overall conductivity was between $104.43 \pm 2.68 \mu \mathrm{S} \mathrm{cm} \mathrm{cm}^{-1}$ and $133.13 \pm 0.95 \mu \mathrm{S} \mathrm{cm}^{-1}$. All sampling sites were normal for general water resources, meaning that the water was livable for living organisms and suitable for human consumption as it did not exceed the quality standard $\left(<300 \mu \mathrm{S} \mathrm{cm}^{-1}\right)$ of surface water. Conductivity is a quality parameter that is used to assess water status. The range for oligomesotrophic status is $50-100 \mu \mathrm{S} \mathrm{cm}^{-1}$, with $100-250 \mu \mathrm{S} \mathrm{cm}^{-}$ 1 for mesotrophic (Shekha et al. 2017). Value of the dissolved oxygen (DO) was between $2.73 \pm 1.08 \mathrm{mg} \mathrm{L}^{-1}$ and $8.60 \pm 0.35 \mathrm{mg} \mathrm{L}^{-1}$. The highest and lowest values were found during the cool dry season at sites 1 and 3 and at site 5 during the rainy season, respectively. All sampling sites met the standard of surface water quality for general water resources (2 $\mathrm{mg} \mathrm{L}^{-1}$ ) (Simachaya 2000). The BOD was between $1.87 \pm 1.50$ and $11.53 \pm 1.29 \mathrm{mg} \mathrm{L}^{-1}$. The highest value occurred during the rainy season at site 2 , while the lowest was recorded in the cool dry season at site 1 . Results showed that the standard of surface water was not exceeded (Simachaya 2000). Amounts of nutrients, such as nitrate nitrogen, ammonium nitrogen and soluble reactive phosphorus, were between $0.5 \pm 0.29$ and $6.4 \pm 0.75 \mathrm{mg} \mathrm{L}^{-1}$, $0.23 \pm 0.03$ and $0.87 \pm 0.32 \mathrm{mg} \mathrm{L}^{-1}$, and $0.35 \pm 0.07$ and $4.21 \pm 1.32 \mathrm{mg} \mathrm{L}^{-1}$, respectively. The highest value occurred during the rainy season, while the lowest was recorded in the cool dry season. The levels of nitrate nitrogen and ammonium nitrogen found at all sites did not exceed the values of Thailand's prescribed surface water quality standards (Simachaya 2000).

Table 2. Distribution and abundance (\%) of phytoplankton in the Kok River at each sampling site during the three seasons

\begin{tabular}{|c|c|c|c|c|}
\hline \multirow{2}{*}{ Taxonomic categories } & \multirow{2}{*}{$\begin{array}{c}\text { Site } \\
\text { distribution }\end{array}$} & \multicolumn{3}{|c|}{ Abundance (\%) } \\
\hline & & Cool dry & Summer & Rainy \\
\hline \multicolumn{5}{|l|}{ Phylum Cyanobacteria/Class Cyanophyceae /Order Synechococcales } \\
\hline \multicolumn{5}{|l|}{ Family Merismopediaceae } \\
\hline Aphanocapsa sp. & $1,2,3$ & 0.9 & 0.0 & 0.3 \\
\hline \multicolumn{5}{|l|}{ Family Coelosphaeriaceae } \\
\hline Coelomoron pusillum (Van Goor) Komárek & $1,2,3,4,5$ & 0.2 & 0.8 & 2.1 \\
\hline \multicolumn{5}{|l|}{ Family Merismopediaceae } \\
\hline Merismopedia punctata Meyen & 3,5 & 0.0 & 0.2 & 0.1 \\
\hline \multicolumn{5}{|l|}{ Family Leptolyngbyaceae } \\
\hline Planktolyngbya contorta (Lemmermann) Anagnostidis \& Komárek & $1,3,5$ & 0.0 & 0.0 & 0.1 \\
\hline \multicolumn{5}{|l|}{ Family Pseudanabaenaceae } \\
\hline Pseudanabaena sp. & 4 & 0.0 & 0.0 & 2.4 \\
\hline \multicolumn{5}{|l|}{ Order Nostocales/Family Aphanizomenonaceae } \\
\hline Cylindrospermopsis raciborskii (Woloszyńska) Seenayya \& Subba Raju & $1,3,4$ & 0.0 & 0.0 & 0.5 \\
\hline \multicolumn{5}{|l|}{ Phylum Chlorophyta/Class Chlorophyceae/Order Chlamydomonadales } \\
\hline \multicolumn{5}{|l|}{ Family Chlamydomonadaceae } \\
\hline Chlamydomonas gloeopara Rodhe \& Skuja & 3,5 & 0.0 & 0.1 & 0.0 \\
\hline \multicolumn{5}{|l|}{ Family Goniaceae } \\
\hline Gonium pectosale O.F.Müller & $2,3,4,5$ & 1.1 & 0.0 & 0.0 \\
\hline \multicolumn{5}{|l|}{ Family Volvocaceae } \\
\hline Eudorina elegans Ehrenberg & 1,3 & 0.3 & 0.3 & 0.5 \\
\hline Pandorina morum (O.F.Müller) Bory & $1,2,3,5$ & 0.0 & 1.6 & 2.0 \\
\hline
\end{tabular}


Order Sphaeropleales/Family Hydrodictyaceae

Pediastrum duplex var. subgranulosum Raciborski

Pediastrum simplex var. simplex Meyen

Class Trebouxiophyceae/Order Chlorellales/Family Chlorellaceae

Actinastrum hantzchii Lagerheim

Chlorella sp.

Dictyosphaerium granulatum Hindák

Dictyosphaerium tetrachotomum Printz

Micractinium quadrisetum (Lemmermann) G.M.Smith

Family Oocystaceae

Crucigeniella crucifera (Wolle) Komárek

Nephrocytium limneticum (G.M.Smith) G.M.Smith

Order Sphaeropleales/Family Neochloridaceae

Golenkinia sp.

Family Selenastraceae

Ankistrodesmus bibraianus (Reinsch) Korshikov

Ankistrodesmus spiralis (W.B.Turner) Lemmermann

Family Selenastraceae

Kirchneriella lunaris (Kirchner) Möbius

Family Scenedesmaceae

Coelastrum astroideum De Notaris

Coelastrum reticulatum (Dangeard) Senn

Coelastrum cf. verrucosum (Reinsch) Reinsch

Comasiella arcuata var. platydisca (G.M.Smith) E.Hegewald \& M.Wolf

Dimorphococcus lunatus A.Braum

Desmodesmus opoliensis (P.G.Richter) E.Hegewald

Tetradesmus acuminatus (Lagerheim) M.J.Wynne

Class Zygnematophyceae/Order Desmidiales/Family Closteriaceae

Closterium parvulum Nägeli

Family Desmidiaceae

Cosmarium contractum var. contractum Kirchner

Cosmarium askeasyi Schmidle

Euastrum turneri W.West

Staurastrum cf. longbrachiatum (Borge) Gutwinski

Phylum Euglenozoa/Class Euglenophyceae/Order Euglenida/Family Phacidae Phacus longicauda (Ehrenberg) Dujardin

Phylum Bacillariophyta/Class Bacillariophyceae/Order Bacillariales/Family Bacillariaceae

Nitzschia palea (Kützing) W.Smith

Nitzschia inconspicua Grunow

Order Cocconeidales Family Achnanthidiaceae

Achnanthidium straubianum (Lange-Bertalot) Lange-Bertalot

Family Cocconeidaceae

Cocconeis pediculus Ehrenberg

Order Cymbellales/Family Cymbellaceae

Cymbella tugidula Grunow

Cymbella tumida (Brébisson) Van Heurck

Family Gomphonemataceae Gomphonema lagenula Kützing

Gomphonema pumilum (Grunow) E.Reichardt \& Lange-Bertalot

Order Fragilariales/Family Fragilariaceae

Synedra ulna (Nitzsch) Ehrenberg

Order Mastogloiales/Family Achnanthaceae

Achnanthes inflata (Kützing) Grunow

Order Naviculales/Family Naviculaceae

Gyrosigma scalproides (Rabenhorst) Cleve

Gyrosigma spenceri (Bailey ex Quekett) Griffith \& Henfrey

Navicula cincta (Ehrenberg) Ralfs

Navicula germainii J.H.Wallace

Order Surirellales/Family Surirellaceae

Surirella sp.

Order Thalassiophysales/Family Catenulaceae

Amphora sp.

Class Coscinodiscophyceae/Order Melosirales/Family Melosiraceae

Melosira varians C.Agardh

Class Mediophyceae/Order Stephanodiscales/Family Stephanodiscaceae

Cyclotella meneghiniana Kützing
$3,4,5$

$3,4,5$

$3,4,5$

$\begin{array}{ll}3,4,5 & 0.0 \\ 3,4,5 & 3.3\end{array}$

$3,4,5 \quad 0.2$

$3,4,5 \quad 2.0$

$3,4,5 \quad 0.5$

$3,4,5$

3,5

0.0

0.0

$1,2,3,4 \quad 0.6$

3

0.0

$1,2,3,4,5 \quad 0.9$

$3,4,5$

$1,2,3,4,5$

2.2

$3,4,5$

$3,4,5$

1,5

$3,4,5$

$3,4,5$

$1,2,3,4,5 \quad 1.2$

2, 3

5

5

$2,3,4,5$

3.1

1.2

0.1

3.4

0.0

0.5

1.7

0.9

0.0

4.5

0.4

5.0

3.4

0.7

2.0

0.3

1.8

0.0

0.6

0.0

1.6

0.4

0.0

0.9

0.0

2.2

0.6

3.1

2.7

0.1

1.5

0.4

4.8

2.0

0.9

0.0

0.8

0.2

0.0

2.0

$\begin{array}{ll}4,5 & 0.2\end{array}$

0.0

$2,4,5$

$3,4,5$

0.0

$1,2,3,4,5 \quad 7.4$

3,4

0.5

$1,2,3,4,5 \quad 6.9$

$1,2,3,4,5 \quad 5.2$

$1,2,3,4,5 \quad 6.8$

$1,2,3,4,5$

1.2

$1,2,3,4,5 \quad 5.5$

0.9

0.1

1.1

1.1

0.7

6.1

0.7

6.5

6.6

3.3

10.4

12.5

2.6

1.1

2.2

3.7

$2,3,4$

0.0

0.0

$1,2,3,4,5 \quad 3.0$

3.4

$1,2,3,4,5 \quad 1.2$

$1,2,3,4,5 \quad 12.7$

2.2

1.1

$\begin{array}{llll}1,2,3,4,5 & 5.3 & 3.8 & 2.8\end{array}$

$\begin{array}{llll}1,2,3,4,5 & 4.4 & 2.9 & 2.9\end{array}$

$2,5 \quad 0.9$

0.7

0.0

$1,2,3,4,5 \quad 3.0$

3.4

4.2

$1,2,3,4,5 \quad 3.7$

3.9

3.9 
Table 3. Distribution and abundance (\%) of benthic diatoms in the Kok River at each sampling site during the three seasons

\begin{tabular}{|c|c|c|c|c|}
\hline \multirow{2}{*}{ Taxonomic categories } & \multirow{2}{*}{$\begin{array}{c}\text { Site } \\
\text { distribution } \\
\end{array}$} & \multicolumn{3}{|c|}{ Abundance (\%) } \\
\hline & & Cool dry & Summer & Rainy \\
\hline \multicolumn{5}{|l|}{ Phyla Bacillariophyta } \\
\hline \multicolumn{5}{|l|}{ Order Bacillariales, Family Bacillariaceae } \\
\hline Nitzschia amphibia Grunow & $1,3,4,5$ & 1.1 & 0.3 & 0.9 \\
\hline Nitzschia inconspicua Grunow & $1,2,3,4,5$ & 6.2 & 6.1 & 3.8 \\
\hline Nitzschia intermedia Hantzsch & $1,2,3,4,5$ & 0.6 & 0.6 & 0.2 \\
\hline Nitzschia palea (Kützing) W.Smith & $1,2,3,4,5$ & 2.5 & 7.4 & 9.7 \\
\hline Nitzschia supralitorea Lange-Bertalot & $1,2,3,4,5$ & 7.2 & 4.0 & 6.0 \\
\hline Tryblionella jelineckii (Grunow) Mann & 3 & 0.2 & 0.2 & 0.2 \\
\hline \multicolumn{5}{|l|}{ Order Cocconeidales, Family Achnanthidiaceae } \\
\hline Achnanthidium pseudoconspicuum var. yomensis Yana \& Mayama & $1,2,3,4,5$ & 1.4 & 2.1 & 0.9 \\
\hline Achnanthidium straubianum (Lange-Bertalot) & $1,2,3,4,5$ & 43.7 & 14.9 & 14.0 \\
\hline Planothidium frequentissimum (Lange-Bertalot) Lange-Bertalot & $1,2,3,4,5$ & 5.4 & 7.0 & 4.7 \\
\hline Planothidium rostratum (Østrup) Lange-Bertalot & $1,2,3,4,5$ & 0.8 & 0.9 & 1.1 \\
\hline \multicolumn{5}{|l|}{ Order Cocconeidales, Family Cocconeidaceae } \\
\hline Cocconeis placentula Ehrenberg & $1,2,3,4,5$ & 4.8 & 12.1 & 1.1 \\
\hline \multicolumn{5}{|l|}{ Order Cymbellales, Family Cymbellaceae } \\
\hline Cymbella tugidula Grunow & $1,2,3,4,5$ & 0.8 & 2.8 & 2.4 \\
\hline Cymbella tumida (Brébisson) Van Heurck & $1,3,4,5$ & 0.1 & 0.1 & 0.4 \\
\hline \multicolumn{5}{|l|}{ Order Cymbellales, Family Gomphonemataceae } \\
\hline Gomphonema lagenula Kützing & $1,2,3,4,5$ & 3.7 & 5.8 & 13.3 \\
\hline Gomphonema pumilum var. rigidum E.Reichardt \& Lange-Bertalot & $1,2,3,4,5$ & 0.7 & 4.0 & 1.0 \\
\hline Gomphonema subclavatum (Grunow) Grunow & $3,4,5$ & 0.0 & 0.2 & 0.1 \\
\hline \multicolumn{5}{|l|}{ Order Fragilariales, Family Fragilariaceae } \\
\hline Fragilaria capucina Desmazières & $1,2,3,4,5$ & 0.1 & 2.7 & 0.1 \\
\hline \multicolumn{5}{|l|}{ Order Licmophorales, Family Ulnariaceae } \\
\hline Ulnaria lanceolata (Kützing) Compère & $1,3,4,5$ & 0.1 & 0.2 & 0.8 \\
\hline \multicolumn{5}{|l|}{ Order Naviculales, Family Diadesmidaceae } \\
\hline Diadesmis confervacea Kützing & $1,2,3,4,5$ & 2.4 & 1.4 & 0.7 \\
\hline Luticola mutica (Kützing) D.G.Mann & $1,2,3,4,5$ & 1.3 & 1.6 & 7.2 \\
\hline \multicolumn{5}{|l|}{ Order Naviculales, Family Naviculales incertae sedis } \\
\hline Mayamaea agrestis (Hustedt) Lange-Bertalot & $1,2,3,4,5$ & 0.5 & 0.5 & 8.1 \\
\hline \multicolumn{5}{|l|}{ Order Naviculales, Family Naviculaceae } \\
\hline Navicula cincta (Ehrenberg) Ralfs & $1,2,3,4,5$ & 1.0 & 2.4 & 1.0 \\
\hline Navicula cryptotenella Lange-Bertalot & $1,3,4,5$ & 0.0 & 0.5 & 0.1 \\
\hline Navicula germainii J.H.Wallace & $1,2,3,4,5$ & 1.4 & 3.5 & 1.6 \\
\hline Navicula simulata Manguin & $1,2,3,4,5$ & 2.0 & 1.9 & 1.7 \\
\hline Navicula sp.1 & $1,2,3,4,5$ & 1.1 & 0.1 & 0.4 \\
\hline Navicula sp. 2 & $1,2,3,4,5$ & 2.3 & 0.7 & 1.0 \\
\hline Navicula $\mathrm{sp} .3$ & $1,2,3,4,5$ & 0.1 & 0.8 & 0.3 \\
\hline Naviculadicta nanogomphonema Lange-Bertalot \& U.Rumrich & $1,2,3,4,5$ & 1.0 & 0.1 & 3.2 \\
\hline Seminavis strigosa (Hustedt) Danieledis \& Economou-Amilli & $1,2,3,4,5$ & 1.1 & 6 & 1.1 \\
\hline Fallacia sp. 1 & 3 & 0.3 & 0.1 & 0.1 \\
\hline Fallacia sp.2 & $3,4,5$ & 0.3 & 0.0 & 0.3 \\
\hline Sellaphora lanceolata D.G.Mann \& S.Droop & $1,2,3,4,5$ & 0.1 & 0.0 & 1.4 \\
\hline Sellaphora pupula (Kützing) Mereschkovsky & $1,2,3,4,5$ & 0.2 & 1.6 & 0.5 \\
\hline Sellaphora sp.1 & $1,2,3,4,5$ & 1.7 & 0.5 & 5.7 \\
\hline Surirella fonticola Hustedt & $2,3,4$ & 0.0 & 0.5 & 0.1 \\
\hline Amphora montana Krasske & $1,2,3,4,5$ & 1.8 & 0.3 & 3.6 \\
\hline Amphora pediculus (Kützing) Grunow & $1,2,3,4,5$ & 2.2 & 5.9 & 1.3 \\
\hline Cyclotella meneghiniana Kützing & $2,3,4,5$ & 0.3 & 0.3 & 0.2 \\
\hline
\end{tabular}

Note: Data are expressed as mean \pm standard deviation (SD) of four replicates. Different letters (a, b and c) represent statistical comparisons between groups in each row using ANOVA and Tukey's post hoc test $(p<0.05)$

The trophic status of the Kok River water was classified as mesotrophic to meso-eutrophic. Results showed that all sampling sites during the cool dry and summer seasons were classified as mesotrophic, while during the rainy season all sites were classified as meso-eutrophic. This result concurred with Pekthong (2008) who indicated that water qualities in Kok River were mesotrophic status in most months, with the exception of the rainy season when water quality changed to meso-eutrophic status. The physical and chemical parameters among the sampling sites showed that conductivity, BOD, nitrate nitrogen, ammonium nitrogen and soluble reactive phosphorus had highest values in the rainy season and lowest values in the cool dry season. 
Table 4. Physical and chemical parameters of water quality (average) in Kok River, Chiang Rai Province, Thailand

\begin{tabular}{|c|c|c|c|c|c|c|c|c|c|c|c|c|c|c|c|}
\hline \multirow[t]{2}{*}{ Parameters } & \multicolumn{5}{|l|}{ Cool dry } & \multicolumn{5}{|l|}{ Summer } & \multicolumn{5}{|l|}{ Rainy } \\
\hline & Site 1 & Site 2 & Site 3 & Site 4 & Site 5 & Site 1 & Site 2 & Site 3 & Site 4 & Site 5 & Site 1 & Site 2 & Site 3 & Site 4 & Site 5 \\
\hline $\begin{array}{l}\text { Water temp. } \\
\left({ }^{\circ} \mathrm{C}\right)\end{array}$ & $22.00 \pm 0.00^{\mathrm{b}}$ & $22.70 \pm 0.58^{b}$ & $22.67 \pm 0.29^{b}$ & $20.67 \pm 0.29^{\mathrm{a}}$ & $22.17 \pm 0.29^{b}$ & $27.00 \pm 0.00^{c}$ & $26.00 \pm 0.00^{c}$ & $26.17 \pm 0.29^{c}$ & $26.00 \pm 0.00^{c}$ & $26.33 \pm 0.58^{c}$ & $26.03 \pm 0.25^{c}$ & $25.97 \pm 0.06^{\mathrm{c}}$ & $25.17 \pm 0.15^{\mathrm{bc}}$ & $24.63 \pm 0.06^{\mathrm{bc}}$ & $26.00 \pm 0.58^{c}$ \\
\hline $\begin{array}{l}\text { Air temp. } \\
\left({ }^{\circ} \mathrm{C}\right)\end{array}$ & $22.00 \pm 0.00^{\mathrm{a}}$ & $25.33 \pm 0.58^{\mathrm{b}}$ & $23.87 \pm 1.76^{\mathrm{ab}}$ & $22.33 \pm 0.29^{\mathrm{a}}$ & $25.00 \pm 0.00^{\mathrm{b}}$ & $31.00 \pm 0.00^{c}$ & $30.00 \pm 0.00^{c}$ & $26.33 \pm 0.58^{b}$ & $26.33 \pm 0.58^{b}$ & $25.67 \pm 1.15^{\mathrm{b}}$ & $27.00 \pm 0.00^{\mathrm{bc}}$ & $30.00 \pm 0.00^{c}$ & $28.00 \pm 0.00^{\mathrm{bc}}$ & $25.00 \pm 0.00^{\mathrm{b}}$ & $28.53 \pm 1.27^{\mathrm{bc}}$ \\
\hline $\begin{array}{l}\text { Velocity } \\
\left(\mathrm{m} \mathrm{s}^{-1}\right)\end{array}$ & $0.25 \pm 1.10^{\mathrm{ab}}$ & $0.30 \pm 1.50^{\mathrm{ab}}$ & $0.23 \pm 1.01^{\mathrm{ab}}$ & $0.36 \pm 0.57^{\mathrm{ab}}$ & $0.14 \pm 0.20^{\mathrm{a}}$ & $0.16 \pm 0.65^{\mathrm{a}}$ & $0.25 \pm 1.74^{\mathrm{ab}}$ & $0.15 \pm 0.29^{\mathrm{a}}$ & $0.29 \pm 1.90^{\mathrm{ab}}$ & $0.12 \pm 0.06^{\mathrm{a}}$ & $0.26 \pm 0.50^{\mathrm{ab}}$ & $0.28 \pm 2.48^{\mathrm{ab}}$ & $0.20 \pm 0.38^{\mathrm{a}}$ & $0.46 \pm 3.21^{\mathrm{b}}$ & $0.18 \pm 0.20^{\mathrm{a}}$ \\
\hline $\begin{array}{l}\text { Turbidity } \\
\text { (NTU) }\end{array}$ & $31.10 \pm 2.93^{\mathrm{b}}$ & $26.77 \pm 1.91^{\mathrm{ab}}$ & $26.90 \pm 4.45^{\mathrm{ab}}$ & $24.67 \pm 3.99^{\mathrm{ab}}$ & $20.73 \pm 3.55^{\mathrm{a}}$ & $23.73 \pm 2.11^{\mathrm{ab}}$ & $32.23 \pm 3.93^{b}$ & $18.27 \pm 1.05^{\mathrm{a}}$ & $16.73 \pm 0.47^{\mathrm{a}}$ & $17.73 \pm 0.91^{\mathrm{a}}$ & $196.67 \pm 3.06^{\mathrm{d}}$ & $\mathrm{d}^{\mathrm{a}} 537.00 \pm 35.59^{\mathrm{e}}$ & $176.00 \pm 33.18^{\mathrm{d}}$ & $122.67 \pm 5.86^{\mathrm{c}}$ & $103.00 \pm 4.58^{\mathrm{c}}$ \\
\hline pH & $7.10 \pm 0.71^{\mathrm{a}}$ & $6.42 \pm 1.50^{\mathrm{a}}$ & $6.42 \pm 0.07^{\mathrm{a}}$ & $6.55 \pm 0.12 \mathrm{a}$ & $6.43 \pm 0.10^{\mathrm{a}}$ & $7.33 \pm 0.15^{\mathrm{a}}$ & $7.27 \pm 0.15^{\mathrm{a}}$ & $7.23 \pm 0.15^{\mathrm{a}}$ & $7.23 \pm 0.06^{\mathrm{a}}$ & $7.17 \pm 0.31^{\mathrm{a}}$ & $7.23 \pm 0.21^{\mathrm{a}}$ & $6.97 \pm 0.15^{\mathrm{a}}$ & $7.10 \pm 0.10^{\mathrm{a}}$ & $7.20 \pm 0.10^{\mathrm{a}}$ & $7.03 \pm 0.15^{\mathrm{a}}$ \\
\hline $\begin{array}{l}\text { Alkalinity } \\
\left(\mathrm{mg} \mathrm{L}^{-1}\right)\end{array}$ & $56.67 \pm 4.16^{\mathrm{a}}$ & $67.30 \pm 1.15^{\mathrm{b}}$ & $68.67 \pm 3.06^{\mathrm{b}}$ & $80.00 \pm 5.29^{c}$ & $72.00 \pm 3.46^{\mathrm{bc}}$ & $125.33 \pm 2.31^{c}$ & $130.67 \pm 15.14^{\text {cd }}$ & $133.33 \pm 18.04^{\text {cd }}$ & $134.67 \pm 6.11^{\mathrm{cd}}$ & $142.67 \pm 4.62^{\mathrm{d}}$ & $140.00 \pm 2.00^{\mathrm{d}}$ & $121.33 \pm 1.15^{\mathrm{c}}$ & $132.00 \pm 5.29^{\mathrm{cd}}$ & $123.33 \pm 3.06^{\mathrm{c}}$ & $133.33 \pm 5.77^{\mathrm{cd}}$ \\
\hline $\begin{array}{l}\text { Conductivity } \\
\left.) \mu \mathrm{S} \mathrm{cm}^{-1}\right)^{*}\end{array}$ & $107.70 \pm 0.30^{\mathrm{a}}$ & $104.77 \pm 6.88^{\mathrm{a}}$ & $104.57 \pm 1.78^{\mathrm{a}}$ & $104.43 \pm 2.68^{\mathrm{a}}$ & $109.27 \pm 4.48^{\mathrm{a}}$ & $123.10 \pm 0.95^{\mathrm{ab}}$ & $129.37 \pm 1.22^{\mathrm{ab}}$ & $126.40 \pm 0.62^{\mathrm{ab}}$ & $131.93 \pm 10.46^{\mathrm{b}}$ & $132.10 \pm 0.87^{\mathrm{b}}$ & $133.13 \pm 0.95^{b}$ & b $117.60 \pm 0.53^{\text {ab }}$ & $112.03 \pm 1.70^{\mathrm{a}}$ & $117.47 \pm 14.01^{\mathrm{ab}}$ & $120.63 \pm 6.47^{\mathrm{ab}}$ \\
\hline $\begin{array}{l}\text { DO } \\
\left(\mathrm{mg} \mathrm{L}^{-1}\right)^{*}\end{array}$ & $8.60 \pm 0.00^{c}$ & $8.30 \pm 0.27^{\mathrm{c}}$ & $8.60 \pm 0.35^{\mathrm{c}}$ & $7.27 \pm 0.31^{\mathrm{bc}}$ & $7.40 \pm 0.53^{\mathrm{bc}}$ & $6.87 \pm 0.31^{\mathrm{b}}$ & $6.67 \pm 0.23^{\mathrm{b}}$ & $6.27 \pm 0.42^{\mathrm{ab}}$ & $5.47 \pm 0.23^{\mathrm{a}}$ & $6.20 \pm 0.53^{\mathrm{ab}}$ & $3.27 \pm 0.06^{\mathrm{a}}$ & $3.20 \pm 0.00^{\mathrm{a}}$ & $3.10 \pm 0.20^{\mathrm{a}}$ & $3.03 \pm 0.21^{\mathrm{a}}$ & $2.73 \pm 1.08^{\mathrm{a}}$ \\
\hline $\begin{array}{l}\mathrm{BOD}_{5} \\
\left(\mathrm{mg} \mathrm{L}^{-1}\right)^{*}\end{array}$ & $1.87 \pm 1.50^{\mathrm{a}}$ & $6.90 \pm 0.64^{\mathrm{b}}$ & $5.27 \pm 0.81^{\mathrm{ab}}$ & $4.53 \pm 1.14^{\mathrm{ab}}$ & $3.2 \pm 0.72^{\mathrm{ab}}$ & $5.20 \pm 1.20^{\mathrm{ab}}$ & $10.93 \pm 0.83^{\mathrm{c}}$ & $6.53 \pm 3.61^{\mathrm{b}}$ & $5.87 \pm 0.23^{\mathrm{ab}}$ & $6.80 \pm 2.43^{\mathrm{b}}$ & $6.93 \pm 1.21^{\mathrm{b}}$ & $11.53 \pm 1.29^{\mathrm{c}}$ & $10.73 \pm 1.03^{\mathrm{c}}$ & $11.40 \pm 1.97^{\mathrm{c}}$ & $10.13 \pm 4.31^{\mathrm{bc}}$ \\
\hline $\begin{array}{l}\text { Nitrate } \\
\text { nitrogen } \\
\text { )mg L-1 })^{*}\end{array}$ & $0.5 \pm 0.29^{\mathrm{a}}$ & $0.8 \pm 0.59^{\mathrm{a}}$ & $0.8 \pm 0.35^{\mathrm{a}}$ & $0.6 \pm 0.20^{\mathrm{a}}$ & $1.0 \pm 0.06^{\mathrm{a}}$ & $0.5 \pm 0.15^{\mathrm{a}}$ & $0.7 \pm 0.35^{\mathrm{a}}$ & $1.4 \pm 1.01^{\mathrm{a}}$ & $1.3 \pm 0.32^{\mathrm{a}}$ & $0.8 \pm 0.20^{\mathrm{a}}$ & $3.3 \pm 0.67^{\mathrm{a}}$ & $4.8 \pm 0.65^{\mathrm{ab}}$ & $4.1 \pm 0.74^{\mathrm{ab}}$ & $6.1 \pm 1.56^{\mathrm{b}}$ & $6.4 \pm 0.75^{\mathrm{b}}$ \\
\hline $\begin{array}{l}\text { Ammonium } \\
\text { nitrogen } \\
\text { )mg L L-1)* }\end{array}$ & $0.46 \pm 0.25^{\mathrm{ab}}$ & $0.84 \pm 0.24^{\mathrm{b}}$ & $0.30 \pm 0.03^{\mathrm{a}}$ & $0.23 \pm 0.03 \mathrm{a}$ & $0.33 \pm 0.15^{\mathrm{a}}$ & $0.39 \pm 0.06^{\mathrm{a}}$ & $0.37 \pm 0.02^{\mathrm{a}}$ & $0.48 \pm 0.33^{\mathrm{a}}$ & $0.68 \pm 0.58^{\mathrm{ab}}$ & $0.35 \pm 0.03^{\mathrm{a}}$ & $0.35 \pm 0.09^{\mathrm{a}}$ & $0.87 \pm 0.32^{\mathrm{b}}$ & $0.55 \pm 0.20^{\mathrm{ab}}$ & $0.63 \pm 0.16^{\mathrm{ab}}$ & $0.29 \pm 0.08^{\mathrm{a}}$ \\
\hline $\begin{array}{l}\text { Soluble } \\
\text { reactive } \\
\text { phosphorus } \\
\left(\mathrm{mg} \mathrm{L}^{-1}\right)^{*}\end{array}$ & $0.53 \pm 0.07^{\mathrm{a}}$ & $0.54 \pm 0.14^{\mathrm{a}}$ & $0.61 \pm 0.28^{\mathrm{a}}$ & $0.35 \pm 0.07^{\mathrm{a}}$ & $0.56 \pm 0.09^{\mathrm{a}}$ & $0.57 \pm 0.28^{\mathrm{a}}$ & $0.79 \pm 0.15^{\mathrm{a}}$ & $0.75 \pm 0.23^{\mathrm{a}}$ & $0.67 \pm 0.12^{\mathrm{a}}$ & $0.71 \pm 0.15^{\mathrm{a}}$ & $0.79 \pm 0.27^{\mathrm{a}}$ & $3.52 \pm 0.54^{\mathrm{b}}$ & $3.86 \pm 0.08^{\mathrm{b}}$ & $4.21 \pm 1.32^{\mathrm{b}}$ & $4.03 \pm 0.79^{\mathrm{b}}$ \\
\hline Trophic status & Mesotrophic & Mesotrophic & Mesotrophic & Mesotrophic & Mesotrophic & Mesotrophic & Mesotrophic & Mesotrophic & Mesotrophic & Mesotrophic & $\begin{array}{l}\text { Meso - } \\
\text { eutrophic }\end{array}$ & $\begin{array}{l}\text { Meso - } \\
\text { eutrophic }\end{array}$ & $\begin{array}{l}\text { Meso - } \\
\text { eutrophic }\end{array}$ & $\begin{array}{l}\text { Meso - } \\
\text { eutrophic }\end{array}$ & $\begin{array}{l}\text { Meso - } \\
\text { eutrophic }\end{array}$ \\
\hline
\end{tabular}



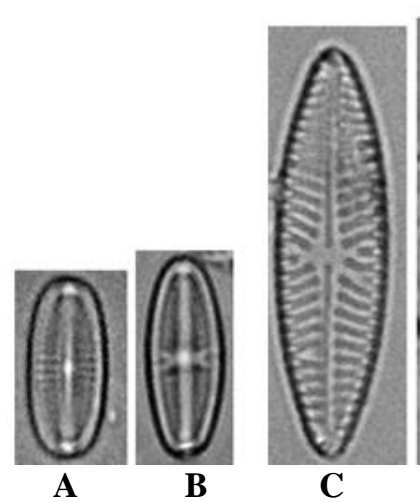

C

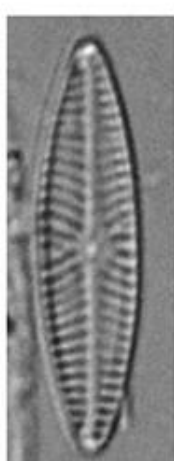

D

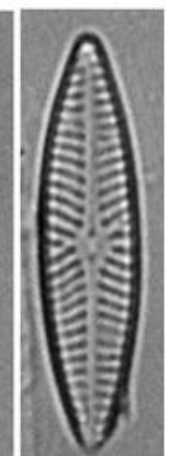

$\mathbf{E}$

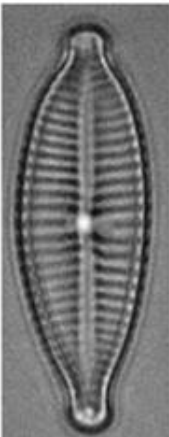

F

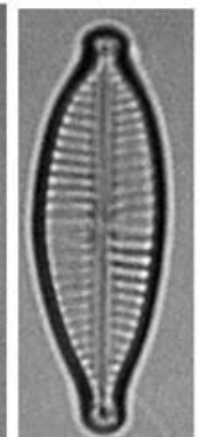

G

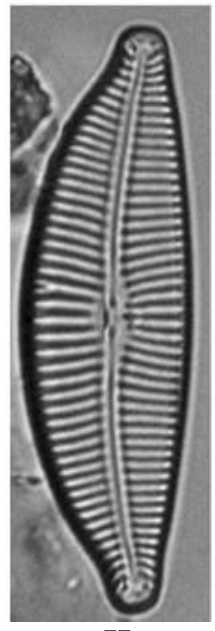

H

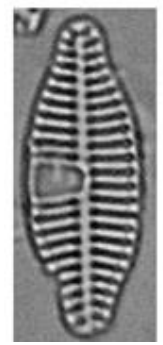

I

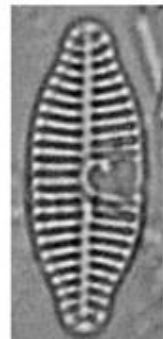

J

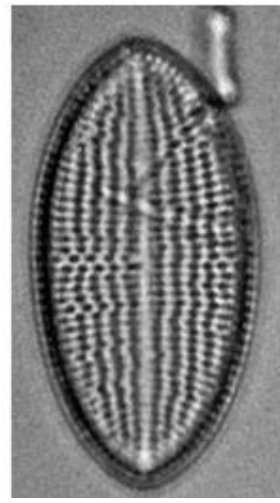

K

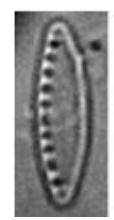

L

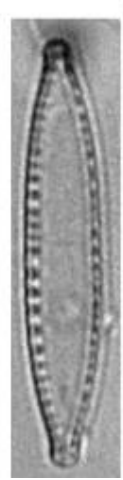

M

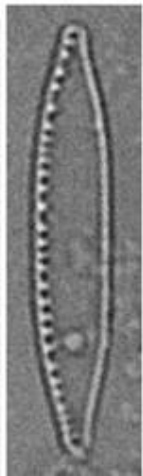

$\mathbf{N}$

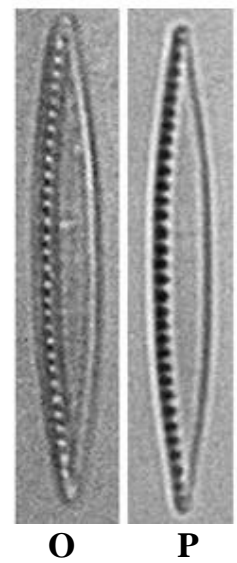

Figure 2. Light micrographs of dominant phytoplankton and benthic diatoms in the Kok River (scale bar $=10 \mu \mathrm{m}$ ). A-B. Achnanthidium straubianum (Lange-Bertalot) ${ }^{*}$, C-E. Navicula cincta (Ehrenberg) Ralfs*, F-G. Gomphonema lagenula Kützing*», H. Cymbella tugidula Grunow*, I-J. Planothidium frequentissimum (Østrup) Lange-Bertalot”, K. Cocconeis placentula Ehrenberg», L. Nitzschia inconspicua Grunow", M-N. Nitzschia palea (Kützing) W.Smith", O-P. Nitzschia supralitorea Lange-Bertalot". Note: The symbol represent to living characteristic: * Phytoplankton, ${ }^{\vee}$ Benthic diatoms and ${ }^{* \boldsymbol{p}}$ Phytoplankton and benthic diatoms

The Kok River is one of the tributaries of the Mekong, and runs for $114 \mathrm{~km}$ across Chiang Rai Province through the general community, restaurants, fish ponds, and agricultural activities. These conditions impact various water quality parameters including nutrient loading (Xu et al. 2015; Withers et al. 2014). Frequent rainfall occurring during June resulted in discharge of nitrogen and phosphorus from the soil and discharge of agricultural fertilizers and wastewater from the community into the water body (Sharpley et al. 2013). Flushing of inorganic nitrogen from $\mathrm{N}$-enriched upper soils horizons has been suggested as the primary mechanism for increasing $\mathrm{N}$ concentrations during rainfall events (Howden et al. 2011). This finding was similar to results reported by Liu et al. (2014), indicating that total nitrogen and nitrate nitrogen under heavy rainfall conditions were higher than measured under conditions of moderate rainfall. Pekthong (2008) found that during the rainy season months, the Kok River water was highly turbid, with high nitrate nitrogen and ammonium nitrogen concentrations.

\section{Correlation between phytoplankton, benthic diatoms and water quality}

Many variables influence the growth of freshwater algae. Environmental factors affect stream conditions and freshwater algal seasonality. Algae possess different physical and chemical requirements, whereby each species has a different set of favorable conditions that promote its growth and reproduction (Prasertsin and Peerapornpisal 2018). Algal communities are sensitive to changes in their environment ( $\mathrm{Li}$ et al. 2018). As a result, algae total biomass and certain designated species are often used as indicators of water quality (Järvinen et al. 2012). In a lotic ecosystem, almost all algae live as benthic forms (Bere and Tundisi 2010).

The relationship between the dominant phytoplankton, benthic diatoms and the physical and chemical characteristics of the water body are shown by the results of the CCA plot (Figure 3). 


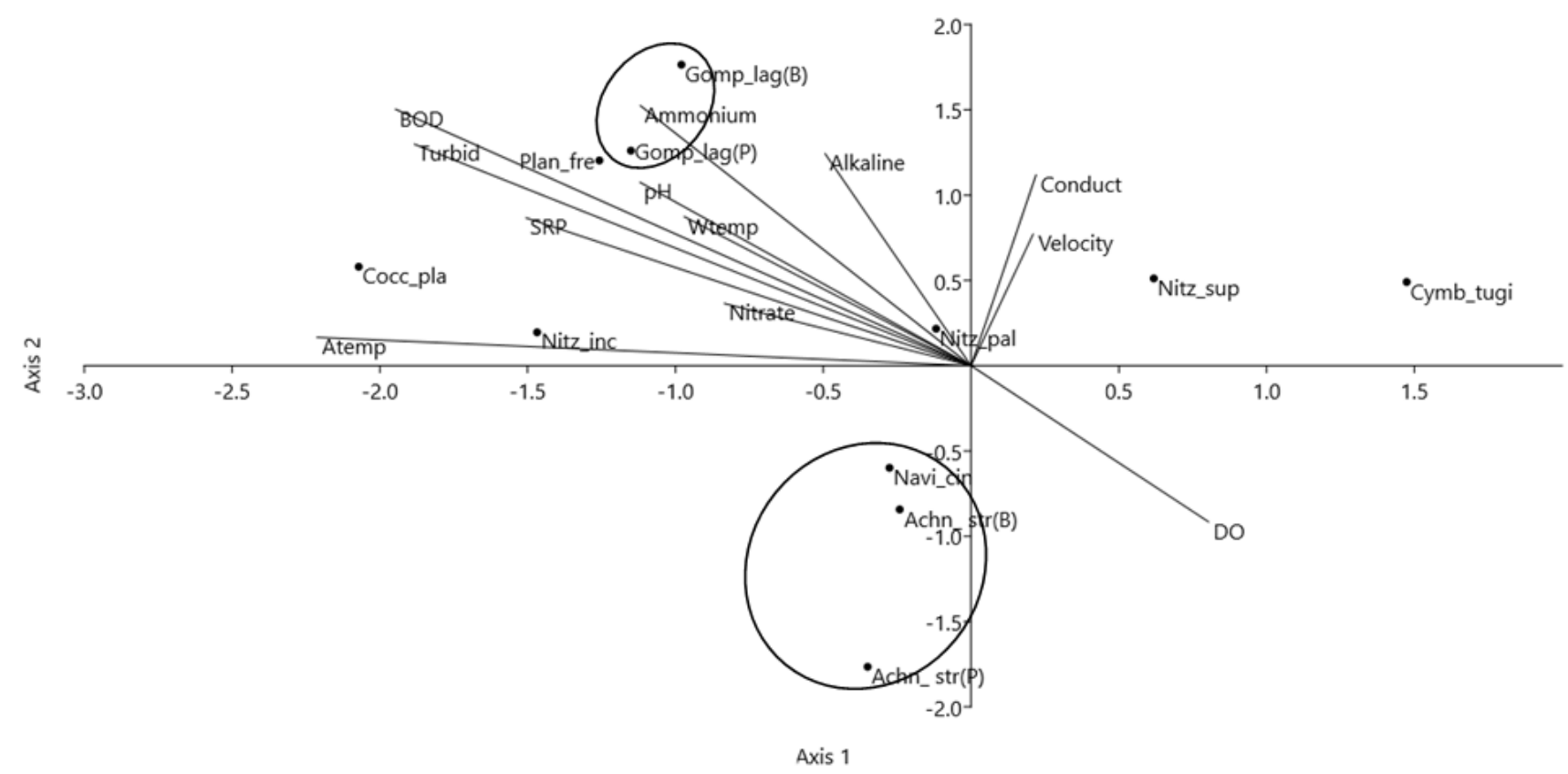

Figure 3. Canonical Correspondence Analysis (CCA) of the physical and chemical parameters and the phytoplankton and benthic diatoms. The results revealed a correlation between the physicochemical parameters and the dominant phytoplankton and benthic diatom in the Kok River, Chiang Rai province, Thailand (Eigenvalues percentage of axis $1=43.2$, axis $=27.62$

Gomphonema lagenula (Gomp_lag) in both planktonic and benthic forms showed a positive correlation with ammonium nitrogen. The highest percentage of abundance of this species was found during the rainy season, with the highest ammonium nitrogen. This result concurred with Leelahakriengkrai and Peerapornpisal (2010) who reported that the species occurred in moderate water quality. Moreover, Achnanthidium straubianum (Achn_str) in both planktonic and benthic forms and Navicula cincta (Navi_cin) showed a negative correlation between velocity and conductivity. The abundance of these species was the lowest during the rainy season with the highest water velocity and conductivity, similar to Ivanov (2018) who reported that these species occurred in moderate water quality.

Nitzschia palea (Nitz_pal) also showed a positive correlation with alkalinity, while Planothidium frequentissimum (Plan_fre) had a positive correlation with pH. Nitzschia inconspicua (Nitz_inc) had a positive correlation with air temperature (Atemp) but a negative correlation with DO (Fig. 3). Temperature was the most important factor controlling which phytoplankton taxa were present in freshwater lakes (Lv et al. 2014). Dominant planktonic and benthic diatoms, such as Nitzschia palea, Gomphonema lagenula and Cocconeis placentula, were good indicator species for moderate water quality. This result was similar to Leelahakriengkrai and Peerapornpisal (2010) findings who reported that these species occurred in moderate water quality.

In conclusion, phytoplankton and benthic diatoms can be found in a wide variety of water qualities that possess different physical and chemical requirements. Water temperature, turbidity, $\mathrm{pH}$, alkalinity, velocity, conductivity, DO, BOD, ammonium nitrogen and soluble reactive phosphorus were the main factors that influenced the composition of phytoplankton and benthic diatoms in Kok River. The combination of phytoplankton and benthic diatom can be used as bio-indicators of water quality in the Kok River and other freshwater ecosystems.

\section{ACKNOWLEDGEMENTS}

The authors acknowledge the National Research Council of Thailand of 2018 for financial support and the Biology Program, Faculty of Science and Technology, Chiang Rai Rajabhat University, to provide laboratory facilities and material support. Finally, we would like to thank Mr. Peter Charge for proofreading the manuscript.

\section{REFERENCES}

Bere T, Tundisi JG. 2010. Biological monitoring of lotic ecosystems: The role of diatoms. Braz J Biol 70 (3): 493-502. DOI: 10.1590/S151969842010005000009

Blanco S, Ector L. 2009. Distribution, ecology and nuisance effects of the freshwater invasive diatom Didymosphenia geminata (Lyngbye) M. Schmidt: a literature review. Nova Hedwigia 88 (3-4): 347-422. DOI: 10.1127/0029-5035/2009/0088-0347

Borics G, Görgényi J, Grigorszky I, László-Nagy Z, Tóthmérész B, Krasznai E, Várbíró G. 2014. The role of phytoplankton diversity metrics in shallow lake and river quality assessment. Ecol Indic 45: 28-36. DOI: 10.1016/j.ecolind.2014.03.011

Eaton AD, Clesceri IS, Rice EW, Greenberg AE. 2005. Standard Method for Examination of Water and Wastewater. $21^{\text {st }}$ ed Virginia. American Public Health Association (APHA), Washington DC. 
Evans AEV, Hanjra MA, Jiang Y, Qadir M, Drechsel P. 2012. Water quality: Assessment of the current situation in Asia. Int J Water Resour Dev 28 (2): 195-216. DOI: 10.1080/07900627.2012.669520

Guiry MD, Guiry GM. 2020. AlgaeBase. Worldwide electronic publication, National University of Ireland, Galway. http://www.algaebase.org. Accessed August 4, 2020.

Howden NJK, Burt TP, Worrall F, Mathias SA, Whelan MJ. 2011. Nitrate pollution in intensively farmed regions: What are the prospects for sustaining high quality groundwater. Water Resour Res 47 (6): W00L02, DOI: 10.1029/2011WR010843.

Ivanov P. 2018. Two new diatom species from family Achnanthidiaceae in Bulgaria: Achnanthidium druartii, an invasive species in Europe and Achnanthidium straubianum, new to Bulgarian diatom flora. Phytol Balc 24 (2): 195-199.

Järvinen M, Drakare S, Free G, Lyche-solheim A, Phillips G, Skjelbred B, Mischke U, Ott I, Poikane S, Søndergaard M, Pasztaleniec A, Van wichelen J, Portielje R. 2012. Phytoplankton indicator taxa for reference conditions in Northern and Central European lowland lakes. Hydrobiologia 704 (1): 97-113. DOI: 10.1007/s10750-012-1315-6

John DM, Whitton BA, Brook AJ. 2011. The Freshwater Algal Flora of the British Isles. Cambridge University Press, Cambridge.

Lange-Bertalot H. 2001. Navicula sensu sticto, 10 genera separated from Navicula sensu lato, Frustulia. In: Lange-Bertalot H (ed) Diatoms of Europe. Diatoms of the European Inland Waters and Comparable Habitats Vol.2. Gantner Verlag ARG, Ruggell KG.

Leelahakriengkrai P, Peerapornpisal Y. 2010. Diversity of benthic diatoms and water quality of the Ping River, Northern Thailand. Environ Asia 3 (1): $82-94$

Leelahakriengkrai P, Peerapornpisal Y. 2011. Diversity of benthic diatoms in six main rivers of Thailand. Int J Agric Biol 13 (3): 309-316.

Leelahakriengkrai P, Peerapornpisal Y. 2014. Evaluation of the trophic benthic diatom index in some main rivers of Thailand. Adv Environ Biol 8 (1): 248-254.

Leelahakriengkrai P, Kunpradid T. 2018. Benthic diatoms in the ping river and its tributaries in Mae Taeng District, Chiang Mai Province, Thailand. Biotropia 25 (3): 181-192.

Liu R, Wang J, Shi J, Chen Y, Sun C, Zhang P, Shen Z. 2014. Runoff characteristics and nutrient loss mechanism from plain farmland under simulated rainfall conditions. Sci Tot Environ 468: 1069-1077. DOI: $10.1016 /$ j.scitotenv.2013.09.035

Li D, Wu N., Tang S, Su G, Li X, Zhang Y, Wang G, Zhang J, Liu H, Hecker M, Giesy JP, Yu H. 2018. Factors associated with blooms of cyanobacteria in a large shallow lake, China. Environ Sci Eur 30 (1): 1-5. DOI: $10.1186 / \mathrm{s} 12302-018-0152-2$

Lobo EA, Heinrich CG, Schuch M, Wetzel CE, Ector L. 2016. Diatoms as Bioindicators in Rivers. In: Necchi O Jr (ed) River Algae. Springer Nature, Cham, Switzerland.

Lv H, Yang J, Liu L, Yu X,Yu Z, Chiang P. 2014. Temperature and nutrients are significant drivers of seasonal shift in phytoplankton community from a drinking water reservoir, subtropical China Environ Sci Pollut Res Int 21 (9): 5917-5928. DOI: 10.1007/s11356014-2534-3

Nakkaew S, Pekkoh J, Peerapornpisal Y. 2015. Diversity of benthic diatoms and relationship with some aspect of water properties in the Wang River, Thailand. Int J Appl Environ Sci 10 (1): 265-280.

Omar WMW. 2010. Perspectives on the use of algae as biological indicators for monitoring and protecting aquatic environments, with special reference to Malaysian freshwater ecosystems. Trop Life Sci Res 21 (2): 51-67.
Peerapornpisal Y, Chaiubol C, Pekkoh J, Kraibut H, Chorum M, Wannathong $\mathrm{P}$, Ngearnpat $\mathrm{N}$, Jusakul $\mathrm{K}$, Thammathiwat A, Chuanunta J, Inthasotti T. 2004. Monitoring of water quality in Ang Kaew Reservoir of Chiang Mai University using phytoplankton as bioindicator from 1995-2002. Chiang Mai J Sci 31 (1): 85-94.

Pekthong T. 2008. The Developing of Trophic Diatom Index for Water Quality Monitoring in Kok River Chiang Rai Province. Mae Fah Luang University, Chiang Rai.

Perumal NV, Rajkumar M, Perumal P, Rajaseka KT. 2009. Seasonal variations of plankton diversity in the Kaduviyar estuary, Nagapattinam, Southeast coast of India. J Environ Biol 30 (6): 10351046.

Phinyo K, Pekkoh J, Pekkoh, Peerapornpisal Y. 2017. Distribution and ecological habitat of Scenedesmus and related genera in some freshwater resources of Northern and North-Eastern Thailand. Biodiversitas 18 (3): 1092-1099. DOI: 10.13057/biodiv/d180329

Pollution Control Department. 2010. Survey, Collection and Analysis of Water Samples and Living Organisms in the Standing Water, Final report, Vol. 2. Ministry of Natural Resources and Environment, Bangkok.

Prasertsin T. Pekkoh J, Pathom-aree W, Peerapornpisal Y. 2014. Diversity, new and rare taxa of Pediastrum spp. in some freshwater resources in Thailand. Chiang Mai J Sci 41 (5.1): 1065-1076.

Prasertsin T, Peerapornpisal Y. 2018. Distribution and isolation of microalgae for lipid production in selected freshwater reservoirs of Northern Thailand. Biodiversitas 19 (1): 343-350. DOI: 10.13057/biodiv/d190147

Sharpley A, Jarvie HP, Buda A, May L, Spears B, Kleinman P. 2013. Phosphorus legacy: Overcoming the effects of past management practices to mitigate future water quality impairment. J Environ Qual 42 (5): 1308-1326. DOI: 10.2134/jeq2013.03.0098

Shekha YA, Ali LAQ, Toma JJ. 2017. Assessment of water quality and trophic status of Duhok Lake Dam. Baghdad Sci J 14 (2): 335-342.

Simachaya W. 2000. Water Quality Management in Thailand. Paper for the Workshop on Environmentally Sound Technology on Water Quality Management: UNEP, Mekong River Commission, November 2000

Stepankova J, Hasler P, Hladka M, Poulickova A. 2012. Diversity and ecology of desmids of peat bogs in the Jeseníky Mts: spatial distribution, remarkable finds. Fottea 12 (1): 111-126.

Suphan S, Peerapornpisal Y. 2010. Fifty-three new record species of benthic diatoms from Mekong River and its tributaries in Thailand. Chiang Mai J Sci 37 (2): 326-343.

Withers PJA, Neal C, Jarvie HP, Doody DG. 2014. Agriculture and eutrophication: Where do we go from here? Sustainability 6 (9): 5853-5875. DOI: 10.3390/su6095853

Xu H, Paerl HW, Qin B, Zhu G, Hall NS, Wu Y. 2015. Determining critical nutrient thresholds needed to control harmful blooms of cyanobacteria in eutrophic lake Taihu, China. Environ Sci Technol 49 (2): 1051-1059. DOI: 10.1021/es503744q

Yana E, Peerapornpisal Y, Mayama S. 2013. Diversity of benthic diatoms and correlation with water quality of Yom River, Thailand. Int J Appl Environ Sci 8 (15): 1935-48.

Yana E, Mayama S. 2015. Two new taxa of Achnanthidium and Encyonema (Bacillariophyceae) from the Yom River, Thailand, with special reference to the areolae occlusions implying ontogenetic relationship. Psychol Res 63 (4): 239-252. DOI: 10.1111/pre.12109

Yang M, Li X, Wang H, Zhang B. 2016. Characteristic of summer microbenthic community in Adjacent sea of Yangtze River Estuary. Stud Mar Sinica 51: 269-278 